\title{
Influence of solvent evaporation rate on crystallization of poly(vinylidene fluoride) thin films
}

\author{
K PRAMOD and R B GANGINENI* \\ Department of Physics, School of Physical Chemical and Applied Sciences, Pondicherry University, Kalapet, \\ Puducherry 605014, India
}

MS received 26 July 2014; accepted 11 November 2014

\begin{abstract}
The processes for obtaining crystalline and smooth poly(vinylidene fluoride) (PVDF) thin films using 2-butanone solvent are explored. The in-situ substrate temperature has been systematically controlled to observe the crystallization process. The in-situ substrate temperature is manipulated to control the rate of evaporation of 2-butanone solvent and is found to have played a vital role in the crystallization of PVDF thin films. Further, X-ray diffraction and Raman microscope were utilized to understand the crystalline phase of PDVF thin films, while atomic force microscopy and scanning electron microscopy have been utilized to investigate the surface morphology and surface roughness of the films.
\end{abstract}

Keywords. Poly(vinylidene fluoride); PVDF thin films; crystallization; phase segregation.

\section{Introduction}

Organic ferroelectric polymers are considered to be promising materials for flexible electronics, and in memory recording industries. ${ }^{1,2}$ Due to their potential device applications and the recent developments in fabrication of nanometre thick films, a sharp rise in this research is noticed. Out of all the polymers, poly(vinylidene fluoride) (PVDF) has been a choice of many researchers due to its pyro, piezo and ferroelectric properties. PVDF is found to exist in $\alpha-, \beta-, \gamma$ - and $\delta$-phases. ${ }^{1,3}$ Out of the four phases, $\beta$-phase is a preferred one as it possesses better ferroelectric and piezoelectric properties. ${ }^{3}$ Many works in the literature are basically concentrated in obtaining the $\beta$-phase PVDF thin films using different solvents, such as dimethyl sulphoxide, dimethylformamide and dimethylacetamide. ${ }^{4-9}$ Very few reports are found on the study of optimization of PVDF thin films using 2-butanone as a solvent. However, obtaining crystalline and smooth thin films with different solvents are found to be still a challenging task. In this study, we have addressed the optimization of crystalline PVDF thin films with 2-butanone solvent, which is prepared using home-built spin coater and further discussed about its crystallization conditions. This piece of work has got started with an aim of obtaining the ferroelectric PVDF thin films for utilizing as a barrier in ferroelectric tunnel junctions.

\footnotetext{
*Author for correspondence (rameshg.phy@ pondiuni.edu.in)
}

\section{Experimental}

PVDF with average-molecular weight of 534000 in powder form with a density of $1.74 \mathrm{~g} \mathrm{ml}^{-1}$ was used for making the solution of PVDF with 2-butanone (Boiling point $\sim 80^{\circ} \mathrm{C}$ ) as a solvent. Both PVDF and 2-butanone were obtained from Sigma-Aldrich with $99.9 \%$ purity. A $7 \mathrm{wt} \%$ solution was prepared and stirred for $24 \mathrm{~h}$ for better uniformity. After stirring, the solution is observed to be in whitish colour. The PVDF thin films were prepared by spin coating method (shown in figure 1).

The films are coated on a clean glass substrate of $1 \mathrm{~cm}^{2}$ size. The spin coater speed has been controlled with a 555 timer using pulse width modulation technique and calibrated the speed with the stroboscopic technique. All the films in this study are deposited on a glass substrate using $7 \mathrm{wt} \%$ PVDF with 2400 r.p.m. for 1 min duration. An in-situ substrate heating facility is developed by means of a halogen lamp for controlling the substrate temperature. The temperature is measured with LM35 sensor and controlled with an intensity regulator. The temperature is calibrated with respect to the distance between the sample and the substrate. The desired temperature can be fixed by adjusting this regulator by reading the corresponding temperature in the LM35 sensor, which was kept very close to the substrate. The setup has a capability to vary the temperature from room temperature to a maximum of $130^{\circ} \mathrm{C}$.

Various approaches have been executed for making smooth and crystalline PVDF thin films. Although several studies have been reported in the literature for obtaining the crystalline films, especially thick crystalline films were made 


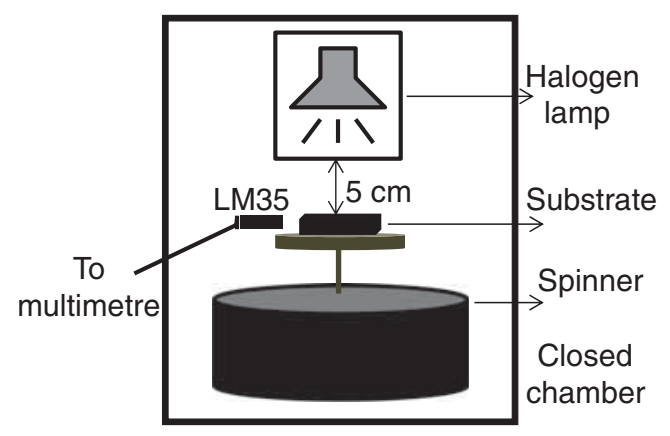

Figure 1. Cartoon picture of homemade spin coater with an in-situ temperature controller attachment.

by controlling the post-annealing temperatures. We have found that obtaining a smooth and crystallized thin film is still a challenging task to achieve and will depend on factors such as humidity and annealing methods. Various optimization (annealling) processes have been considered, which include non-heating method (room temperature deposition, indicated as RT). Another approach followed is to deposit at room temperature and are annealed immediately after the deposition using the halogen lamp at $120^{\circ} \mathrm{C}$ for $20 \mathrm{~min}$ (named as RT120). Both the films deposited at RT and RT120 are annealed for $17 \mathrm{~h}$ at $120^{\circ} \mathrm{C}$ to know whether or not any appearance of crystalline phase with respect to annealing method. The films obtained in this process are named as RTA and RT120A, respectively. However, no significant crystallization peaks are observed in the post-annealing method. Post annealing is found to help the crystallinity of the micrometre thick films and also influences the formation of $\beta$-phase. ${ }^{6}$ Two methods such as Still and Hot (SH) and Rotation and Hot (RH) are considered for further investigation in obtaining the PVDF thin films. Subramaniyan Ramasundaram $e t a l^{4}$ and Mengyuan Li et $_{a l^{7,8}}$ reported that in-situ substrate temperature influences the crystallinity of the PVDF films. In the SH method, the cleaned glass substrate is loaded onto the spinner and heated to the desired temperature with the help of a halogen lamp as shown in figure 1 . Once the steady temperature is reached on the substrate to the desired level, the solution is added onto this SH substrate in such a way that whole substrate surface is covered and further spin coated for 1 min duration at 2400 r.p.m. After the coating, the films were kept for $10 \mathrm{~min}$ for annealing at the same temperature, mainly for the removal of remaining solvent. In this method, several films are deposited at $70,110,120$ and $130^{\circ} \mathrm{C}$ and they are named as SH70, SH110, SH120 and SH130, respectively. The RH method is similar to $\mathrm{SH}$ method except with one difference, the films are prepared when the spin coater is under rotation. The films prepared in this method are termed as RH120. The structural characterizations of the PVDF thin films are carried out with the help of PANalytical X-ray diffraction (XRD) technique. Bruker's MM8 atomic force microscope (AFM) has been used for analysing the surface roughness as well as the microstructure

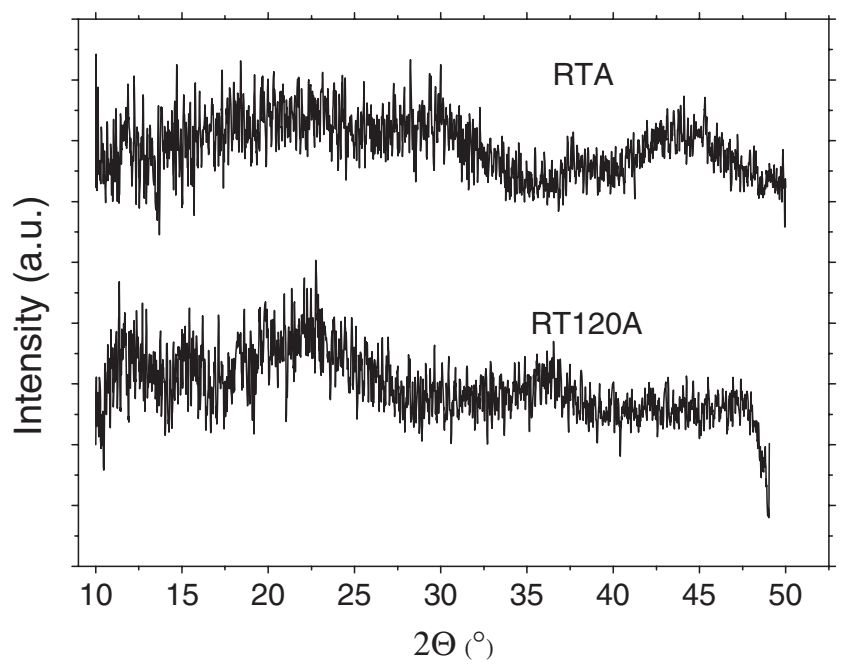

Figure 2. XRD patterns of the films RTA and RT120A. The patterns are moved arbitrarily for clarity.

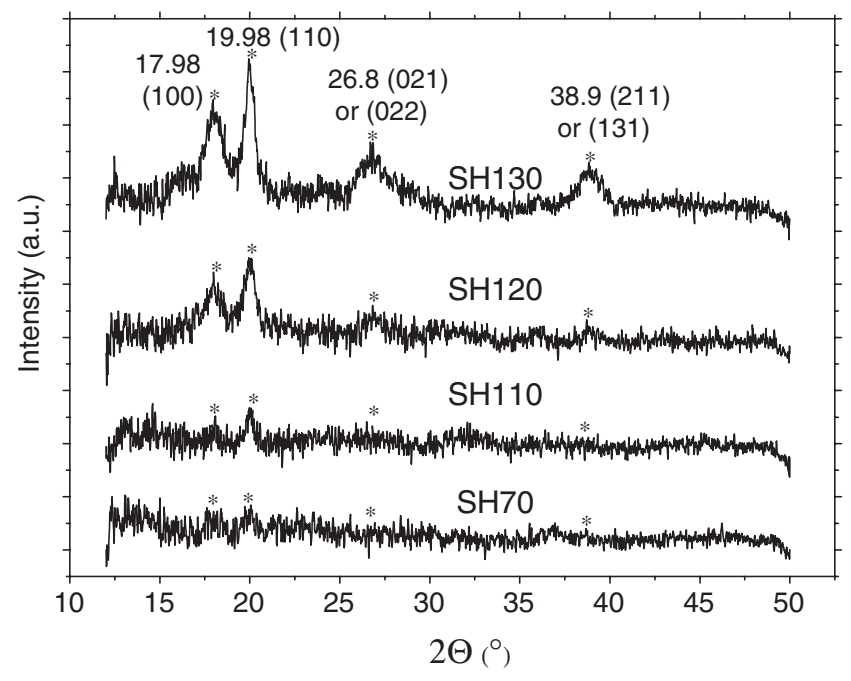

Figure 3. XRD patterns of the films deposited under Still and Hot method at various temperatures from 70 to $130^{\circ} \mathrm{C}$; $\mathrm{SH} 70, \mathrm{SH} 110$, SH120, SH130.

of the PVDF thin films. RenishawInvia Raman microscope $(\lambda=785 \mathrm{~nm})$ was used to correlate the structural characterizations from XRD.

\section{Results and discussion}

XRD patterns of RTA and RT120A films are shown in figure 2. No indication of crystallization peaks is observed and it confirms an amorphous phase formation. This typical amorphous nature is not seen in figure 2 due to the background correction. Further, no change in the XRD patterns is observed after annealing these films at $120^{\circ} \mathrm{C}$ for $17 \mathrm{~h}$ duration.

The XRD patterns of the films prepared under SH method are presented in figure 3 . The in-situ substrate temperature is varied from 70 to $130^{\circ} \mathrm{C}$. The films that are prepared at substrate temperatures of $70^{\circ} \mathrm{C}$ and up to $100^{\circ} \mathrm{C}$ do not 
show any significant crystallization peaks in the XRD image. The films that are deposited at 110,120 and $130^{\circ} \mathrm{C}$ show crystallized peaks. So it is noticed that the crystallization starts above $100^{\circ} \mathrm{C}$. The peaks at $2 \theta$ of $17.98^{\circ}$ and $19.98^{\circ}$ are found to be common in the films of SH110, SH120 and SH130, except with a difference in their peak intensities. The peaks at $2 \theta$ angles of $17.98^{\circ}$ and $19.983^{\circ}$ are more intense in SH130 compared with the SH110 and SH120 films. From the XRD pattern of SH130 film, two major peaks at 26.8 and 38.9 are also identified, whereas these two are very weak in SH110 and SH120 films. By comparing XRD patterns with the existing JCPDS data, it is observed that PVDF thin films are crystallized in $\alpha$-phase. ${ }^{10-13}$ However, from the recent reports on PVDF thin films, it is found that the peak positions are same even for $\gamma$ - and $\delta$-phases except with a difference in the peak intensity. ${ }^{8}$ Thus the exact determination of phase is observed to be difficult with the existing XRD data and its attribution to a particular phase. Raman spectra are used further to understand the structural phase of PVDF thin films. The XRD patterns of films processed under RH method, RH120 and RH120A, show clear crystalline peaks. Because of their non-uniform and crack nature of the films, the films are not considered for further investigation.

Depending on the preparation method, polymers can be crystalline, semicrystalline or amorphous or often they all can be present in a single sample. In semicrystalline state, the regions of aligned chains of small crystallites in a polymer would diffract and remaining chains that have no order lead to incoherent scattering. Thus, the diffraction profile can be understood as a mixture of crystalline diffraction and amorphous scattering nature. It is well understood that highly crystalline polymers (note that highly crystalline polymers also contain quantifiable amount of amorphous content when analysed properly) show sharp well-defined peaks. For semicrystalline polymers (partial crystalline and partial amorphous), broad peaks are observed due to small crystallites and usually the crystalline peaks are visible on a broad hump of amorphous background.

Polymer crystallinity, which indicates the amount of crystalline region with respect to the amorphous content, is an important parameter that determines many of the polymer properties like hardness, stiffness, melting point etc. and most importantly the electroactive properties. In this study, we calculate the $\%$ crystallinity of the PVDF thin films (SH110, 120 and 130) deposited using SH method. In equation 1, the total area in the denominator represents the area of both crystalline and non-crystalline, whereas the numerator indicates the area of crystalline peaks. The area is calculated using peak analyzer available in data analysis and graphing software. An example (SH130) of the fitting procedure and the $\%$ crystallinity calculation is shown in figure 4 . The calculated values are presented in table 1.

$$
\% \text { Crystallinity }=\frac{\text { Total area of crystalline peaks }}{\text { Total area of all peaks }}
$$

The $\%$ crystallinity is found to increase with respect to increase in in-situ substrate temperature. From figures 2 and 3 , it may be said that the in-situ substrate temperature played a vital role in obtaining the crystalline films. The \% crystallinity is found to vary from 7 to $52 \%$ with increase in substrate temperature from 110 to $130^{\circ} \mathrm{C}$.

The thickness of the films is estimated using the tapping mode AFM measurement technique. Figure 5 shows the height image of the film SH120, in which a scratch is made to obtain a step in PVDF films. The step region is scanned using tapping mode AFM. From the step profile image, the thickness is estimated as $275 \mathrm{~nm}$. AFM technique is also used to study the surface morphology of the films.

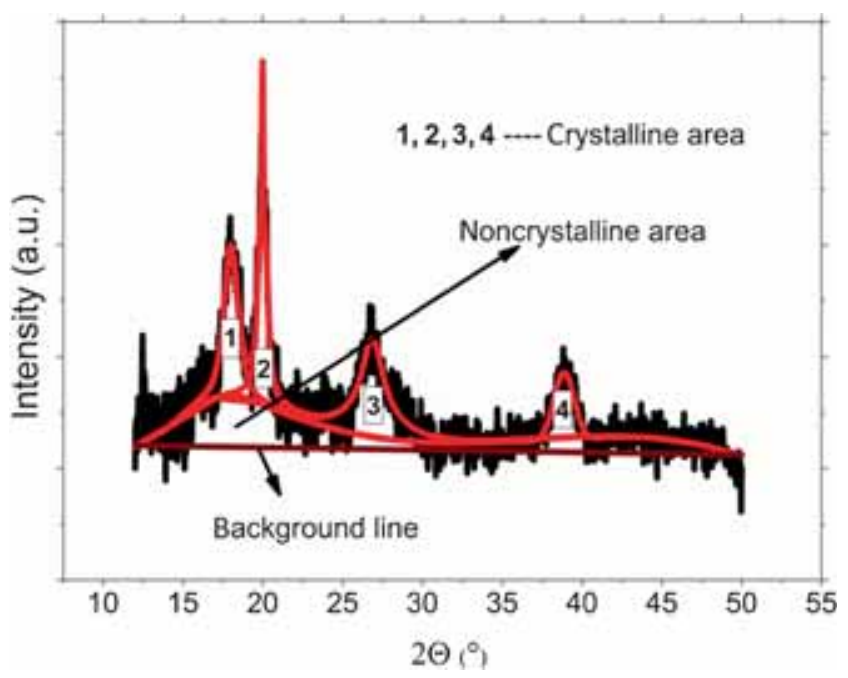

Figure 4. Crystallinity calculation of one of the films deposited under Still and Hot method at $130^{\circ} \mathrm{C}$.

Table 1. Crystallinity and roughness values of the samples prepared under SH deposition method.

\begin{tabular}{lcc}
\hline Sample name & \% Crystallinity & $\begin{array}{c}\text { Rms roughness in an } \\
\text { area of } 1 \mu \mathrm{m}^{2}\end{array}$ \\
\hline SH70 & - & - \\
SH110 & 7 & $9.67 \mathrm{~nm}$ \\
SH120 & 32 & $8.32 \mathrm{~nm}$ \\
SH130 & 52 & $5.75 \mathrm{~nm}$ \\
\hline
\end{tabular}

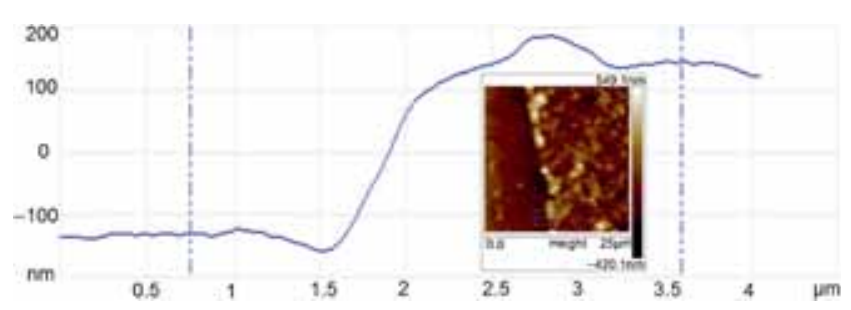

Figure 5. Thickness measurement using AFM technique. A section analysis of the step in AFM height image taken for an area of $25 \mu \mathrm{m}^{2}$ corresponds a thickness of $275 \mathrm{~nm}$. 
(a) $\mathrm{SH} 110$

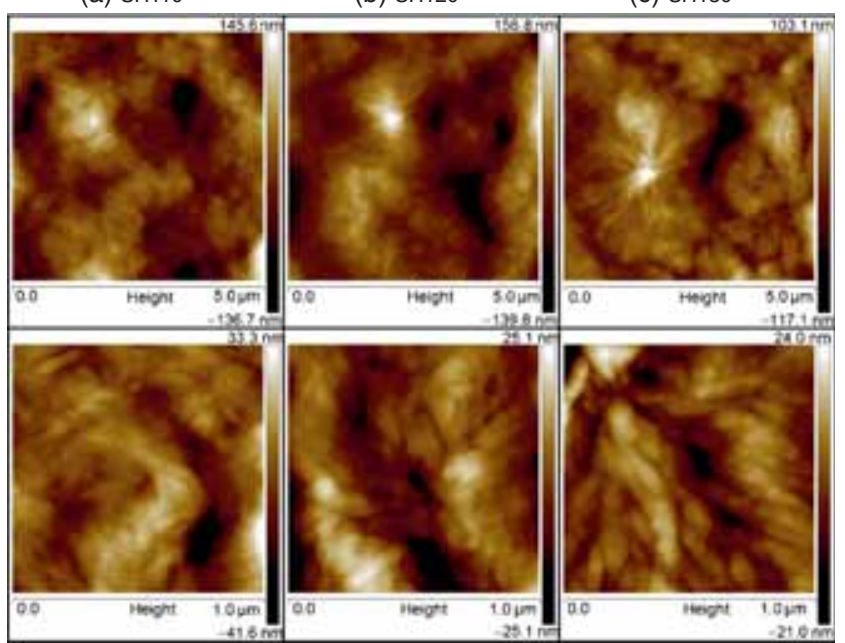

Figure 6. AFM height images of the films deposited by $\mathrm{SH}$ method; SH110, SH120 and SH130 measured in an area of 5 and $1 \mu \mathrm{m}^{2}$.

Figure 6 shows the AFM height images of the samples in $\mathrm{SH}$ category, scanned in an area of 5 and $1 \mu \mathrm{m}^{2}$. To know the quality of the surfaces, rms roughness values are estimated with the help of NanoScope analysis software in an area of $1 \mu \mathrm{m}^{2}$, which are listed in table 1 . From the AFM analysis of the samples RT and RTA (figures are not shown), it is observed that the films are rough in nature and spherical shaped grains are observed. Similarly, the film prepared under RH method shows high roughness of $80 \mathrm{~nm}$, cracks and nonuniform surfaces are observed. Clear formation of elongated grains is observed for SH130 and SH120 samples. In case of $\mathrm{SH} 110$, the formation of elongated grains is not clear. From figure 6 , as we go from 110 to $130^{\circ} \mathrm{C}$, it is interesting to note that elongated grains growth is improved (see images scanned for $1 \mu \mathrm{m}^{2}$ area), accordingly spherulites formation also (see images scanned for $5 \mu \mathrm{m}^{2}$ area). From figure $6 \mathrm{c}$ it is clear that the observed elongated grains are crystalline lamella, grown radially from the centre of the spherulites. Thus crystallinity is an important factor that gives unique property to polymers and is well supported by the formation of lamella at high temperature. Comparing the XRD and AFM images, SH130 show maximum crystallinity of 52\% and the corresponding microstructure shows an elongated type grains. The films deposited below $110^{\circ} \mathrm{C}$ show spherical shaped grains and do not show any crystalline nature. Formation of elongated grains is observed for the films deposited above $110^{\circ} \mathrm{C}$ with SH method. SeokJu Kang et al $l^{5}$ reported that $\beta$-phase PVDF thin films possess elongated grains via rapid thermal annealling. The formation of elongated shaped grains may be due to the transfer of rotational kinetic energy from the spin coater and as well as with respect to the high rate of evaporation. AFM images also show decrease in the rms roughness with increase in the substrate temperature.

Figure 7 shows the AFM phase images of SH samples measured in an area of 5 and $1 \mu \mathrm{m}^{2}$. The map of the phase of the cantilever oscillation is generally used to detect the (a) $\mathrm{SH} 110$

(b) SH120
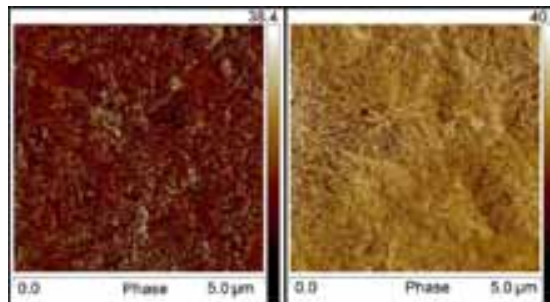

(c) $\mathrm{SH} 130$

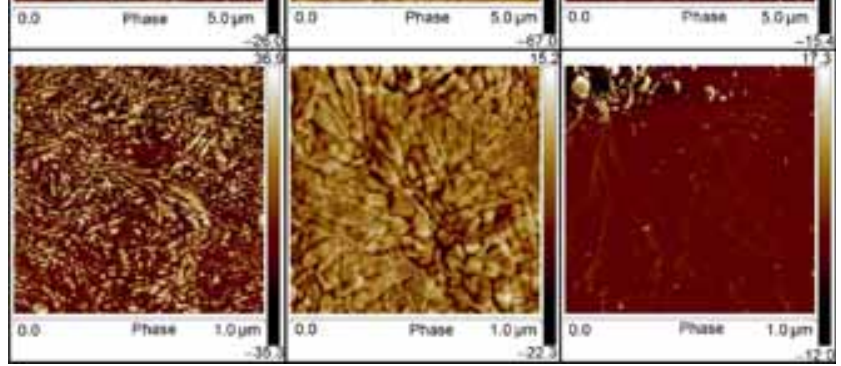

Figure 7. AFM phase images of the films deposited by $\mathrm{SH}$ method; SH110, SH120 and SH130 measured in an area of 5 and $1 \mu \mathrm{m}^{2}$.

microstructural variations in structurally segregated samples, especially in composites. Figure 7a shows the phase image of SH110, which indicates the phase segregation between grains. This phase segregation is possibly due to the change in the structural phase: from the crystalline phase of PVDF grains to the amorphous phase. From figure $7 b$ and $c$, the reduction in the phase segregation is observed. The reduction in the phase segregation is attributed to the increase in the \% crystallinity.

Scanning electron microscopy (SEM) images of SH70, SH120 and SH130 are shown in figure 8. The SEM images reaffirm the formation of spherical grains at $70^{\circ} \mathrm{C}$ and also show the formation of bubbles at higher temperatures 120 and $130^{\circ} \mathrm{C}$. The bubble sizes appeared to be $\sim 90 \mu \mathrm{m}$ diameter in SH120 and in SH130 sample it is noticed to be of $\sim 190 \mu \mathrm{m}$ in diameter. By comparing, XRD, AFM and SEM images, we can conclude that rate of evaporation played a key role in the crystallization process. As the evaporation rate increased with increase in temperature, the bubble sizes also increased. The formation of bubble may be attributed to the miscibility and the wt $\%$ of the solution.

In general, the roughness of the films depends upon many factors such as humidity, substrate temperature, method of preparation, solvent type and substrate roughness. In our method of preparation of the PVDF thin films, the decrease in roughness is observed with increase in the in-situ substrate temperature. The Kardar-Parisi-Zhang proposed a kinetic roughening theory, in which a smooth surface can be obtained at higher temperature due to higher surface diffusibility. ${ }^{14,15}$ Shadowing effect (leads to rough surface) and surface diffusion (leads to smooth surface) effect are the two important factors to decide the surface quality regardless of the preparation methods. Here, the in-situ substrate temperature with its high diffusibility and quick evaporation of butanone solvent may have resulted in obtaining crystalline and smooth PVDF thin films. In addition to these 


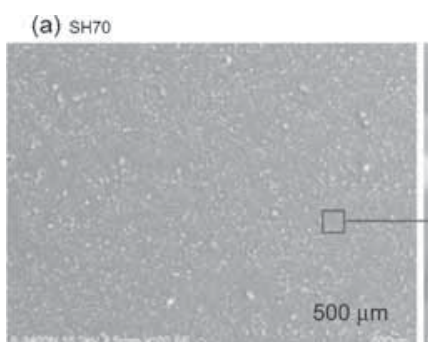

(b) $\mathrm{SH70}$

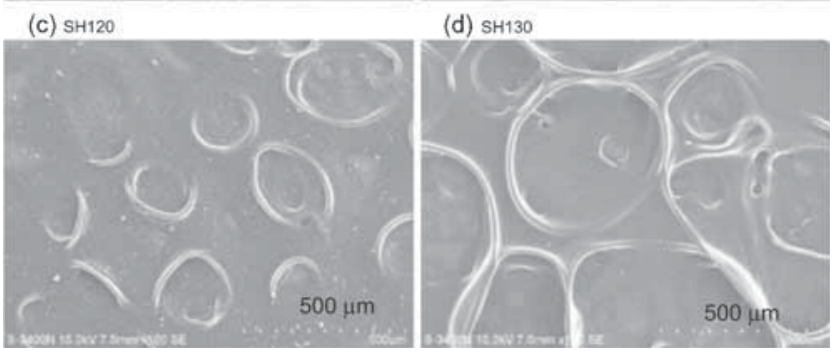

Figure 8. SEM images of the films deposited under $\mathrm{SH}$ method; SH70, SH120 and SH130.

above-mentioned measurements, Raman spectra have been obtained from 300 to $1000 \mathrm{~cm}^{-1}$ using RenishawInvia Raman microscope with $\lambda=785 \mathrm{~nm}$. Figure 9 displays the Raman spectra of the PVDF powder and for the films SH70, SH110, SH120 and SH130.

The PVDF powder Raman spectra indicate a formation of $\alpha$-phase with peaks at 410, 489, 612 and $795 \mathrm{~cm}^{-1}$. As we process the films under $\mathrm{SH}$ method, the peaks appeared predominantly at $795 \mathrm{~cm}^{-1}$ for SH70, SH110 and SH120 samples. The intensity of the peak at $795 \mathrm{~cm}^{-1}$ has increased for SH120 compared with the SH70 and SH110 films spectra. Raman spectra of SH130 indicate the major $\alpha$-phase peaks at $410,489,612$ and $795 \mathrm{~cm}^{-1}$. In addition to the $\alpha$-phase, a small fraction of $\beta$ - and $\gamma$-phase signatures appeared with peaks at 839 and $874 \mathrm{~cm}^{-1}$. Raman images conclude that the films are stabilized in $\alpha$-phase. ${ }^{16-18}$ However, the influence of crystallinity with rise in substrate temperature is noticed with the increase in the intensity of the Raman image. Also, Raman images are in clear agreement with the XRD images.

It is evident from the analysis that the quick rate of evaporation has influenced the formation of a smooth and crystallized $\alpha$-phase PVDF thin films. An oriented (or crystalline) seed grain creation is speculated at temperatures $\geq 110^{\circ} \mathrm{C}$ and this seed grain may have further helped the films to develop crystalline films. The XRD images, Raman microscopy helped in confirming the phase and other existing phases in the PVDF thin films. For low evaporating rates of solvent, the quality and the crystalline nature of the films might be influenced by the humidity $\%$, which may further lead to vapor-induced phase separation, as noticed by Mengyuan Li et al. ${ }^{7}$

Dante Luis Chinaglia et $a l^{9}$ reported that solvent evaporation rate influences the crystalline phases of the solution-cast PVDF films of thickness $>10 \mu \mathrm{m}$. Theoretical simulations would be necessary in understanding the crystallization process and the relation between the substrate temperature,

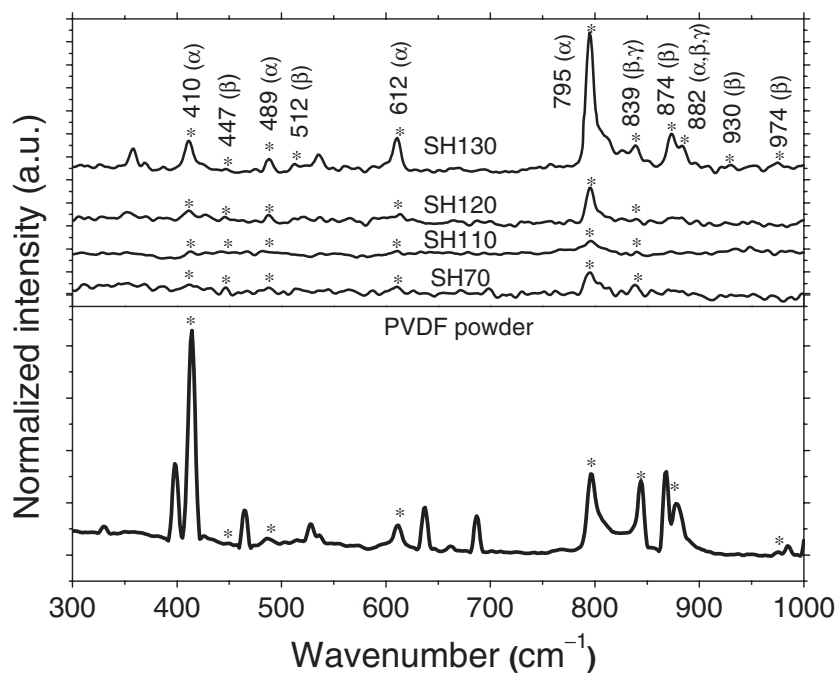

Figure 9. Raman spectroscopy images of the PVDF powder and the thin films of SH70, SH110, SH120 and SH130.

boiling point of the solvent and the miscibility of the solution.

The following points can be concluded from this study:

1. The $\alpha$-phase crystalline PVDF thin films can easily be realized by adjusting the substrate temperatures above the boiling point of the solvent. The quick evaporation rate of the solvent is vital in deciding the roughness, crystalline phase and \% crystallinity of the PVDF thin films.

2. The miscibility and humidity influence on the surface quality of the thin films can also be taken care by the in-situ substrate temperature.

Few setbacks are also found in this process in connection with the films utilization in devices; the PVDF films are crystallized in $\alpha$-phase and for ferroelectric device applications, but $\beta$-phase is preferred. Obtaining the $\beta$-phase and ultrasmooth surfaces is still challenging and it may be crucial to evaporate at even higher substrate temperature or apply an in-situ strain to the films. There are many reports in the literature in turning the thicker films into $\beta$-phase by stretching the films. ${ }^{19}$ However, in thin films, it is still an open question in regard to the application of strain to the films and also to obtain a smooth film surface. Especially in microelectronic applications, there is a clear need of smooth and crystalline thin films to prevent electrical shorts and as well as to have a good contact with the electrodes. This study is able to show that we could optimize the semicrystalline PVDF thin films of thickness of $275 \mathrm{~nm}$ (which is rare for our knowledge with 2-butanone solvent) into a smooth non-polar $\alpha$-phase. Now, the non-polar phase of the PVDF thin films may be converted into polar by the application of an electric pulse to the device or by exposing the film to corona plasma. Second setback is the pre-heated substrate stimulates the bubble formation and this may be avoided with respect to the high rates of evaporation. 
So, this article indeed presents an evidence for the rate of evaporation dependence in obtaining smooth and crystallized PVDF thin films and independent of solvent usage with the condition that the substrate temperature shall be maintained above the boiling point of the solvent.

\section{Conclusion}

A smooth ( $\sim 6 \mathrm{~nm}$ rms roughness) and up to $52 \%$ crystalline $\alpha$-phase PVDF thin films of thickness of $275 \mathrm{~nm}$ are prepared successfully using a homemade spin coater with SH method. The crystallinity appeared above $100^{\circ} \mathrm{C}$ substrate temperature. The crystallinity is attributed to the quick evaporation rates, which achieved due to the boiling point temperature of the solvent. The clear phase segregation in the AFM phase images show an agreement with increase in \% crystallinity with respect to the in-situ substrate temperature. This easy and quick method of preparing smooth and crystalline PVDF thin films using 2-butanone might be useful for the extended experiments to obtain ferroelectric, piezoelectric PVDF thin films.

\section{Acknowledgements}

We would like to acknowledge DAE-BRNS with sanction No: 2012/20/37P/09/BRNS with BSC, UGC-MRP/F.No-41846/2012 (SR), UGC-SAP F.530/15/DRS/2009 and also Central Instrumentation Facility (CIF) at Pondicherry University. K Pramod would like to acknowledge the UGC for Rajiv Gandhi National Fellowship.

\section{References}

1. Andrew J Lovinger 1983 Science 2201115

2. Scott J F 2007 Science 315954
3. Hari Singh Nalwa 1995 Ferroelectric polymers: chemistry physics and applications (New York: Marcel Dekker), XII p 895

4. Subramaniyan Ramasundaram, Sun Yoon, Kap Jin Kim and Jong Soon Lee 2008 Macromol. Chem. Phys. 2092516

5. SeokJu Kang, Youn Jung Park, Jinwoo Sung, Pil Sung Jo, Cheolmin Park, Kap Jin Kim and Beong Ok Cho 2008 Appl. Phys. Lett. 92 012921-1

6. Satapathy S, Santosh Pawar, Gupta P K and Varma K B R 2011 Bull. Mater. Sci. 34727

7. Mengyuan Li, Ilias Katsouras, Claudia Piliego, Gunnar Glasser, Ingo Lieberwirth, Paul W M Blom and Dago M de Leeuw 2013 J. Mater. Chem. C 17645

8. Mengyuan Li, Harry J Wondergem, Mark-Jan Spijkman, Kamal Asadi, Ilias Katsouras, Paul W M Blom and Dago M de Leeuw 2013 Nat. Mater. 12433

9. Dante Luis Chinaglia, Rinaldo Gregorio Jr, Josiani Cristina Stefanello, Ruy Alberto Pisani Altafim, Werner Wirges, Feipeng Wang and Reimund Gerhard 2010 J. Appl. Polym. Sci. 116785

10. Davis G T, McKinney J E, Broadhurst M G and Roth S C 1978 J. Appl. Phys. 494998

11. Gregorio R Jr and Ueno E M 1999 J. Mater. Sci. 344489

12. Rinaldo Gregorio Jr 2006 J. Appl. Polym. Sci. 1003272

13. Ryozo Hasegawa, Yasuhiro Takahashi, Yozo Chatani and Hiroyuki Tadokoro 1972 Polym. J. 3600

14. Mehran Kardar, Giorgio Parisi and Yi-Cheng Zhang 1986 Phys. Rev. Lett. 56889

15. Tzu-Wen Huang, Hsin-Yi Lee, Yung-Wei Hsieh and Chih-Hao Lee 2002 J. Cryst. Growth 237492

16. Boccaccio T, Bottino A, Capannelli G and Piaggio P 2002 Membrane Sci. 210315

17. Masamichi Kobayashi, Kohji Tashiro and Hiroyuki Tadokoro 1975 Macromolecules $\mathbf{8} 158$

18. Shichao Zhang, Juan Shen, Xinping Qiu, Dangsheng Weng and Wentao Zhu 2006 J. Power Sources 153234

19. Salimi A and Yousefi A A 2003 Polym. Test. 22699 